Musées, Patrimoine et Culture scientifiques et techniques

$191 \mid 2020$

septembre-octobre 2020

\title{
Un PSC pour repenser le muséum de Genève. Une démarche originale en Suisse
}

Jacques Ayer

\section{OpenEdition \\ Journals}

Édition électronique

URL : https://journals.openedition.org/ocim/4121

DOI : 10.4000/ocim.4121

ISSN : 2108-646X

Éditeur

OCIM

Édition imprimée

Date de publication : 1 septembre 2020

Pagination : 30-37

ISSN : 0994-1908

Référence électronique

Jacques Ayer, « Un PSC pour repenser le muséum de Genève. Une démarche originale en Suisse », La Lettre de I'OCIM [En ligne], 191 | 2020, mis en ligne le 01 septembre 2021, consulté le 10 décembre 2021. URL : http://journals.openedition.org/ocim/4121 ; DOI : https://doi.org/10.4000/ocim.4121

Ce document a été généré automatiquement le 10 décembre 2021.

Tous droits réservés 


\section{Un PSC pour repenser le muséum de Genève. Une démarche originale en Suisse}

Jacques Ayer

Le projet de nouveau bâtiment du muséum de Genève dédié aux collections est l'un des principaux résultats de la mise en œuvre du PSC.

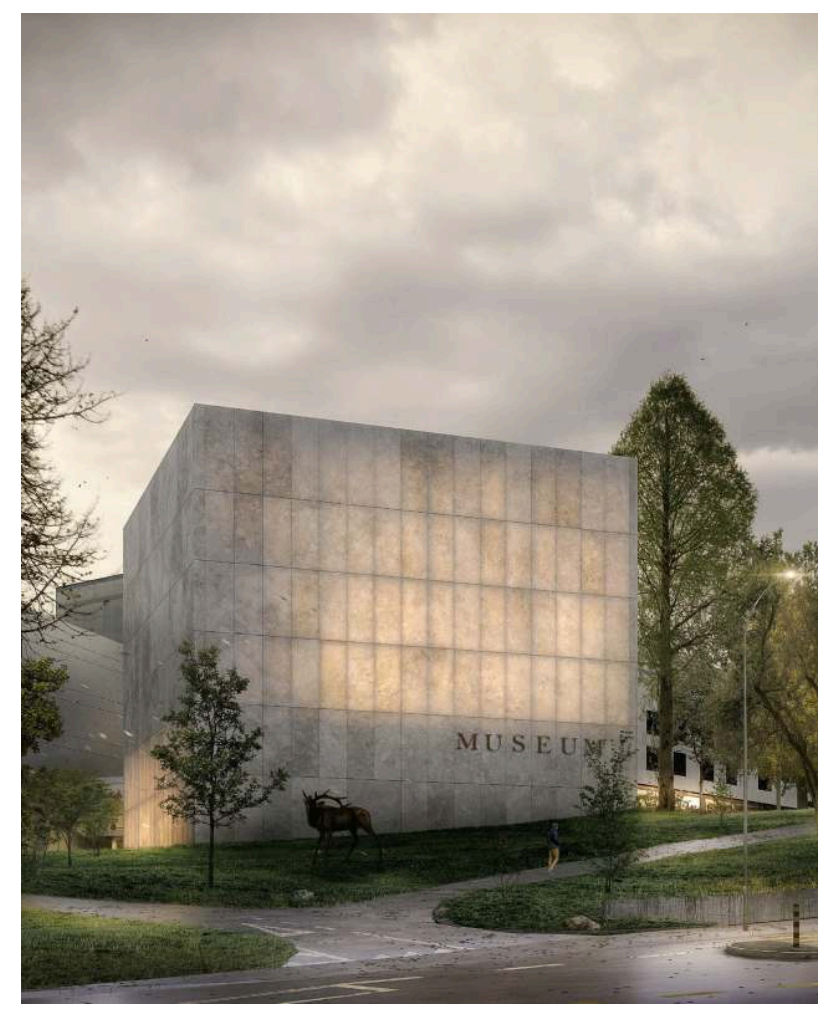

(c) Représentation virtuelle : Bureau MAK architecture SA, lauréat du concours 
indépendante de l'évolution de la société. Dans un monde culturel et technologique en constante mutation marqué par un effondrement environnemental sans précédent et plus récemment par une crise sanitaire historique, on ne souhaite plus que ces institutions s'en tiennent à un rôle unique de conservation, d'étude ou d'éducation. Elles doivent désormais s'interroger sur leurs missions, l'évolution des attentes des publics, leur rôle dans la cité et dans la société, du local à l'international (direction des Musées de France, 2007).

4 Le monde des musées a toujours connu des heures glorieuses mais aussi des périodes de " crises » durant lesquelles les institutions ont dû questionner leur identité, leur vocation et leur positionnement, dans un paysage économique, culturel et social en perpétuel mouvement. Dans le contexte actuel de crise environnementale, les défis à relever n'ont cependant jamais été aussi nombreux que durant cette dernière décennie.

5 En 2012, lors de ma nomination en tant que directeur du muséum de Genève, la tutelle m'a confié la mission principale d'envisager une redynamisation et une redéfinition du rôle et des missions de l'institution tout en tenant compte de ses forces et de ses spécificités.

6 Pour répondre à cette demande et aux nouveaux défis décrits plus haut, j'ai décidé d'opter pour l'élaboration d'un PSC. Cette démarche, totalement nouvelle dans le paysage muséal genevois, a abouti, fin 2014, à la publication d'un rapport assorti d'un plan d'actions permettant d'accompagner le muséum de Genève dans un processus de mutation globale.

Le muséum de Genève occupe le site de Malagnou depuis 1966.

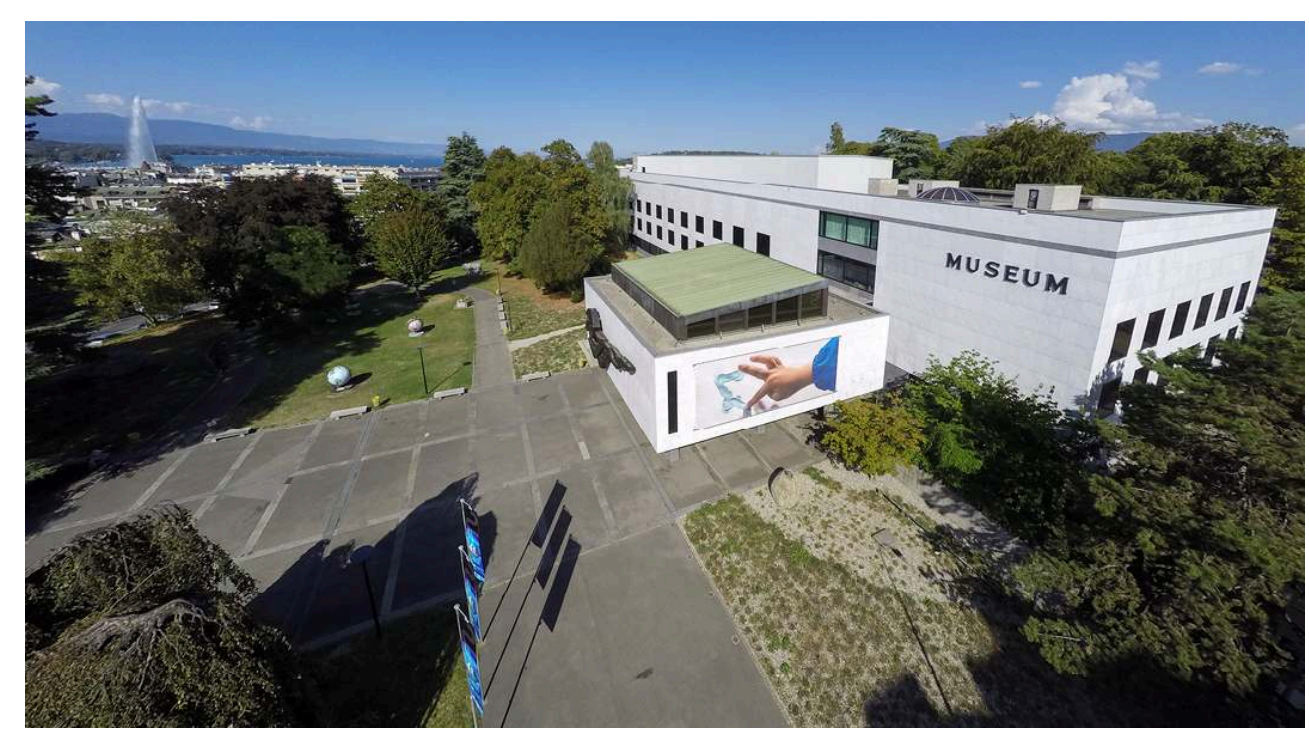

(C) P. Wagneur, MHNG 


\section{Origine et définition du PSC, petit rappel}

7 C'est en France, dans les années 1980, que la démarche du PSC a été initiée par la direction des Musées de France, à l'époque des grands chantiers de rénovation. Cette démarche a été ensuite réglementée par la loi relative aux musées de France du 4 janvier 2002 et ses décrets d'application. La méthode préconisée consiste en un bilan de l'existant assorti d'un diagnostic critique. Puis, un projet sélectif est organisé autour d'une vision globale, présentant des axes stratégiques formalisés par un plan d'action (Joly, 2009).

8 En Suisse, l'établissement de projets scientifiques et culturels est resté jusqu'ici très peu développé. Il n'est pas exigé par les tutelles. Le muséum de Genève est une des premières institutions muséales helvétiques à s'être lancée dans cette démarche.

\section{Le muséum de Genève, une institution bicentenaire}

Comme la plupart des muséums européens, le muséum de Genève est né au début du XIXe siècle d'une mise en commun de cabinets de curiosités initiée par quelques savants genevois. Ces derniers créèrent ainsi un musée académique dont la principale mission était l'enseignement des sciences, de l'histoire et de l'art. Genève est alors encore sous occupation française. Puis, lorsque la cité de Calvin gagne son indépendance, la Ville de Genève décide de racheter les collections du Musée académique et de les rendre accessibles au public dès 1820. Le Musée académique devient alors muséum d'Histoire naturelle.

L'institution va ensuite connaître deux déménagements principaux, l'un en 1872 dans un des bâtiments de l'actuelle université des Bastions, puis en 1965 sur son site actuel de Malagnou.

Le muséum compte aujourd'hui plus de cent collaborateurs représentant 96 postes équivalents plein temps. Sa fréquentation actuelle approche les 300000 visiteurs par an. Mise à part la réintroduction en 2018 d'une billetterie pour les expositions temporaires, l'accès au musée reste totalement gratuit.

\section{De l'évaluation de l'existant à la rédaction du nouveau projet}

12 L'évaluation menée de 2012 à 2014 dans tous les domaines d'activité du muséum a permis d'identifier un certain nombre de forces mais aussi des points d'efforts et des faiblesses. Voici quelques éléments de diagnostic mis en lumière à l'époque.

13 Au niveau des missions de recherche et de gestion des collections, une production scientifique soutenue de niveau international et des collections d'importance mondiale constituent les points forts du muséum depuis plusieurs décennies. Avec près de 15 millions de spécimens, la collection de Genève est la plus riche de Suisse et une des dix plus importantes d'Europe. Les conditions de stockage présentent par contre un point de vigilance important. En effet, les valeurs hygrométriques et thermiques ne répondent plus aux normes de conservation, ce qui a notamment pour conséquence d'exposer les collections à des attaques répétées d'insectes ravageurs. De plus, en 2010, 
une estimation du volume total d'alcool utilisé pour les collections (près de 45000 litres) et la vétusté des installations ont amené les autorités et les spécialistes en protection contre les accidents majeurs à mettre en demeure le muséum au vu du risque significatif d'incendie.

En ce qui concerne l'offre culturelle, plusieurs marges d'amélioration ont été identifiées. Le parcours permanent couvrant près de $8000 \mathrm{~m}^{2}$ d'expositions et caractérisé par une longue suite de dioramas, a été conçu et réalisé il y a plusieurs décennies. Certains secteurs présentent une obsolescence de contenu scientifique et de mise en scène qui ont notamment amené au démontage en 2014 de la partie consacrée à l'histoire de la Terre. Quant aux expositions temporaires, elles souffrent de ne pas disposer d'un espace dédié et suffisant en termes de surface disponible. Leur programmation ne répond à aucune politique claire et cohérente tant au niveau de la périodicité, du contenu que du style scénographique. L'offre de médiation, certes riche et diversifiée, est confrontée à une demande très forte que les ressources humaines internes ne peuvent satisfaire complètement.

$\mathrm{Au}$ niveau organisationnel, l'organigramme structurel est basé essentiellement sur un fonctionnement par métiers et non par projets. Sa lecture est rendue compliquée par la multitude de secteurs d'activité et les trois grandes missions du musée ne sont pas clairement identifiables.

Les ressources financières, quant à elles, au demeurant importantes, sont caractérisées par un fort déséquilibre avec une part salariale représentant près de $90 \%$ du budget total.

Les infrastructures enfin, qui n'ont pas connu de transformation structurelle majeure depuis la construction du muséum sur son site de Malagnou en 1965, nécessitent une rénovation importante, tant au niveau de l'accueil que des services.

18 Ce bilan et diagnostic confronté aux nouveaux défis que doit relever le muséum de Genève a permis de proposer en 2014 une vision ambitieuse, de nouvelles orientations et une série de mesures qui, tout en renforçant les missions de l'institution vers plus de complémentarité, tendent vers une meilleure définition de l'ensemble des activités muséales, dans une trajectoire commune et cohérente. Sept axes de développement déclinés en objectifs stratégiques proposent de nouvelles pistes d'évolution dans un esprit holistique. L'encadré, page suivante résume la vision du PSC et les principaux développements envisagés. Enfin, pour offrir une traduction opérationnelle à ces nouvelles orientations, un plan d'actions est défini avec un certain nombre de mesures complétées par un échéancier. L'ensemble des propositions du PSC font l'objet d'un rapport circonstancié publié fin 2014 (Ayer et al., 2014). 
Installation scénographique originale de l'exposition Prédations (2018) qui invite le public à une réflexion autour de la problématique environnementale de la consommation industrielle de viande.

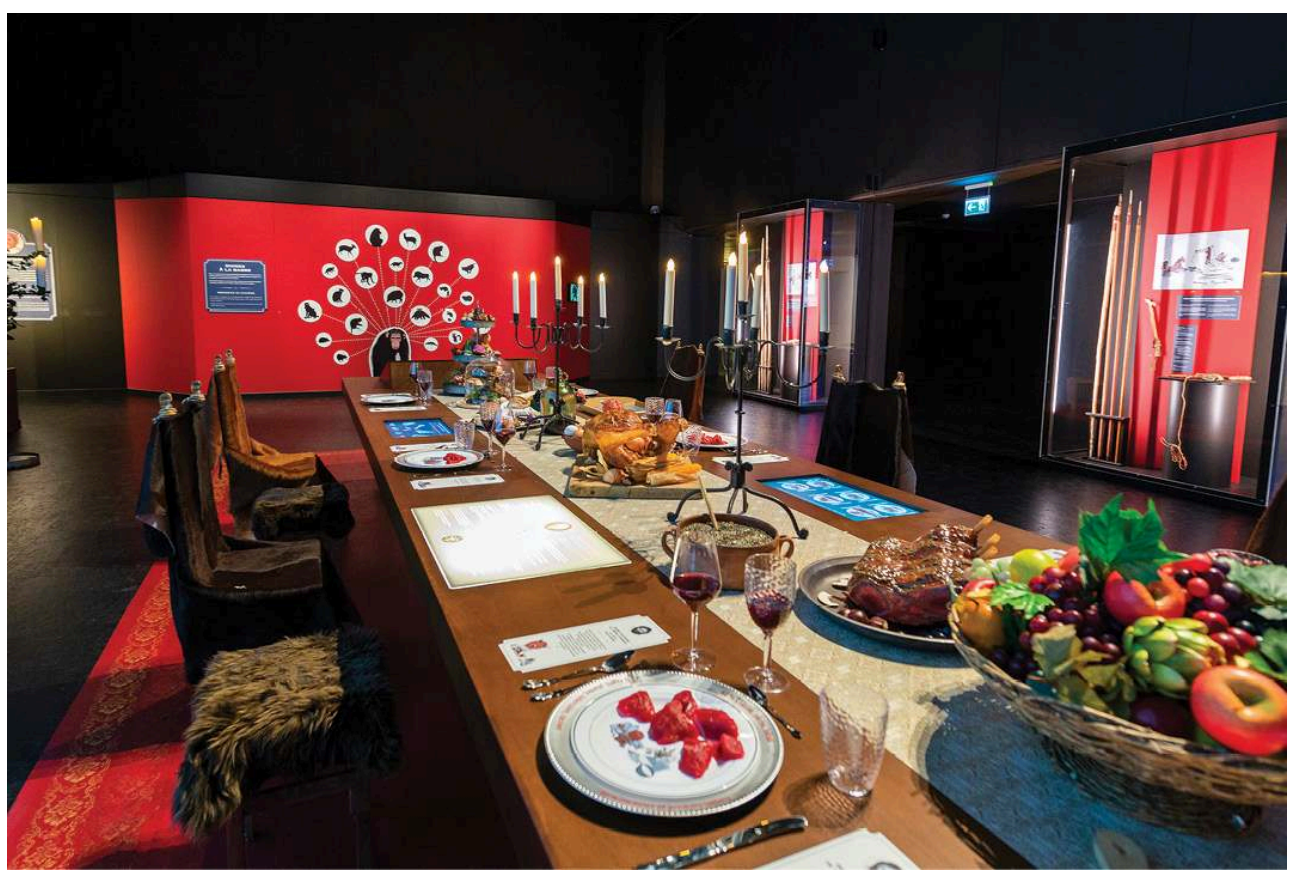

(c) P. Wagneur, MHNG

\section{Premiers résultats} notamment des nouvelles exigences légales internationales et les nouveaux besoins en lien avec la transition écologique. Leur visibilité a été renforcée auprès des publics, notamment adolescents, par la création d'un nouvel espace d'interactivité qui invite le visiteur à découvrir et expérimenter le métier de chercheur au muséum au travers d'un serious game ludique et pédagogique. La notoriété et le rayonnement du muséum ont été fortement étendus lors de l'organisation en 2018 à Genève de la conférence annuelle du plus grand réseau européen de musées et centres scientifiques (Ecsite) qui a fait 
découvrir le muséum à plus de 1200 professionnels provenant de 58 pays différents. Une gouvernance repensée avec l'introduction notamment d'une unité des publics et des expositions a conduit à l'élaboration d'un nouvel organigramme pour accompagner la mise en œuvre du PSC. Ce dernier favorise un fonctionnement par projets et tend vers un meilleur équilibre entre les missions du musée tout en favorisant les échanges et la complémentarité des savoirs et des savoir-faire. En outre, le taux d'absentéisme qui a été réduit de moitié entre 2012 et 2015 est resté stable depuis la mise en œuvre du PSC.

Mais le résultat le plus significatif et prometteur est sans aucun doute le lancement en 2017 d'un projet d'extension et de rénovation partielle du muséum qui offrira des nouvelles infrastructures pour le stockage des collections, mais aussi une réorganisation des espaces de travail et une rénovation des infrastructures d'accueil. En parallèle, le muséum prévoit la rénovation complète de l'exposition permanente consacrée aux sciences de la Terre, l'aménagement d'un espace dédié aux expositions temporaires et la redynamisation des galeries existantes. Ce projet inédit dans l'histoire du muséum est complet et globalisant. C'est une opportunité extraordinaire pour l'institution d'accélérer sa mutation et d'entrer ainsi de plain-pied dans le XXI ${ }^{\mathrm{e}}$ siècle. La votation du budget de réalisation par les autorités politiques pour l'ensemble de l'opération est prévue en 2021. Si le projet est accepté, le chantier devrait être terminé en 2026.

23 La vision et les objectifs du PSC ont fortement contribué à initier ce projet auprès des autorités et des décideurs en démontrant sa pertinence et sa cohérence. En effet, ce dernier répond à la plupart des objectifs du plan d'action.

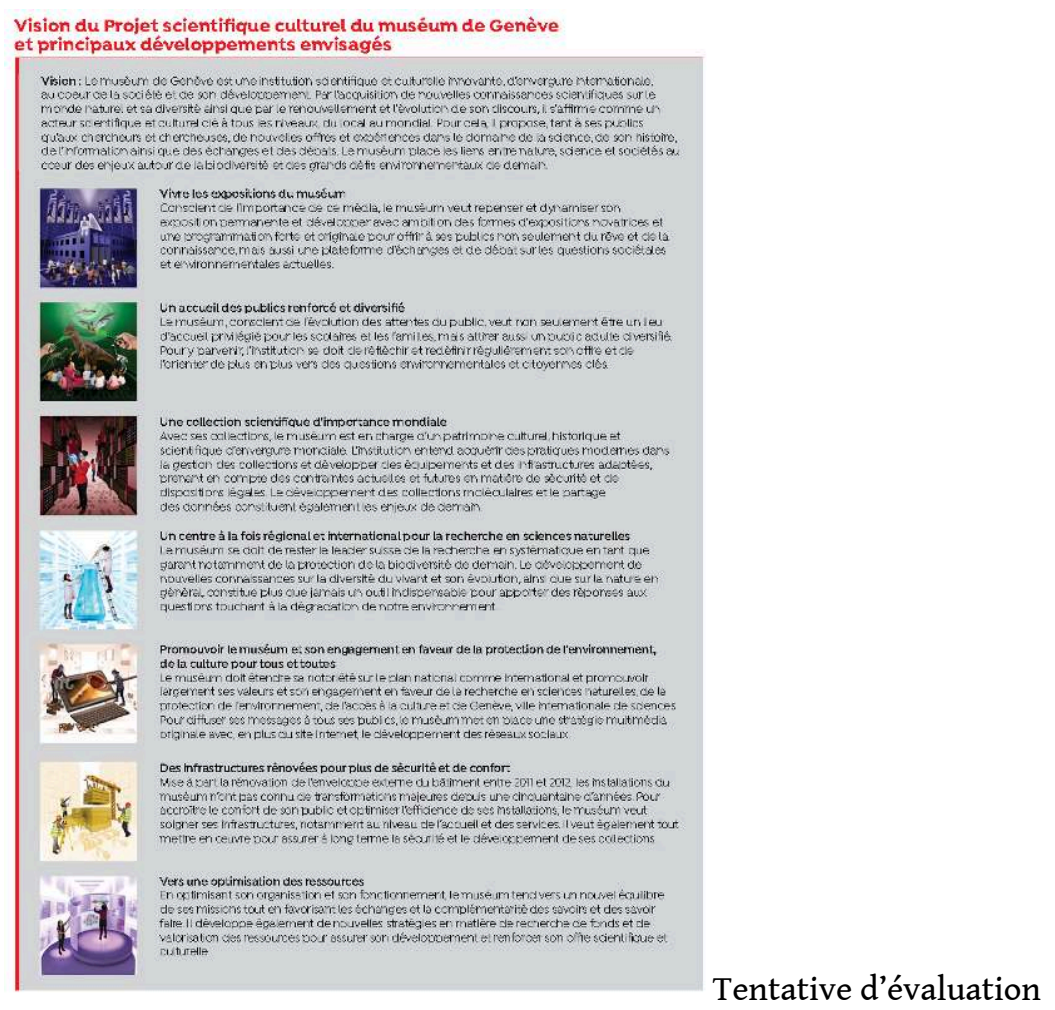

Cinq ans après la mise en œuvre du PSC, l'élaboration d'indicateurs de performance a pour but de fournir un nouvel outil pour le suivi de son développement et une évaluation de sa pertinence. La première étape délicate est de déterminer de bons indicateurs quantitatifs. Leur utilisation soulève immanquablement la question de leur 
représentativité et de leur finalité, car le risque qu'ils soient utilisés comme mesure de rentabilité est bien réel. Cette problématique suscite d'ailleurs un vif débat au sein de la communauté muséale depuis une trentaine d'années (Mairesse, 2004). Dans le cas du PSC du muséum de Genève, ces données ont permis d'identifier les secteurs impactés positivement, mais aussi ceux qui n'ont pas connu d'évolution significative.

Avec 15 millions de spécimens, la collection du muséum de Genève est la plus importante de Suisse.

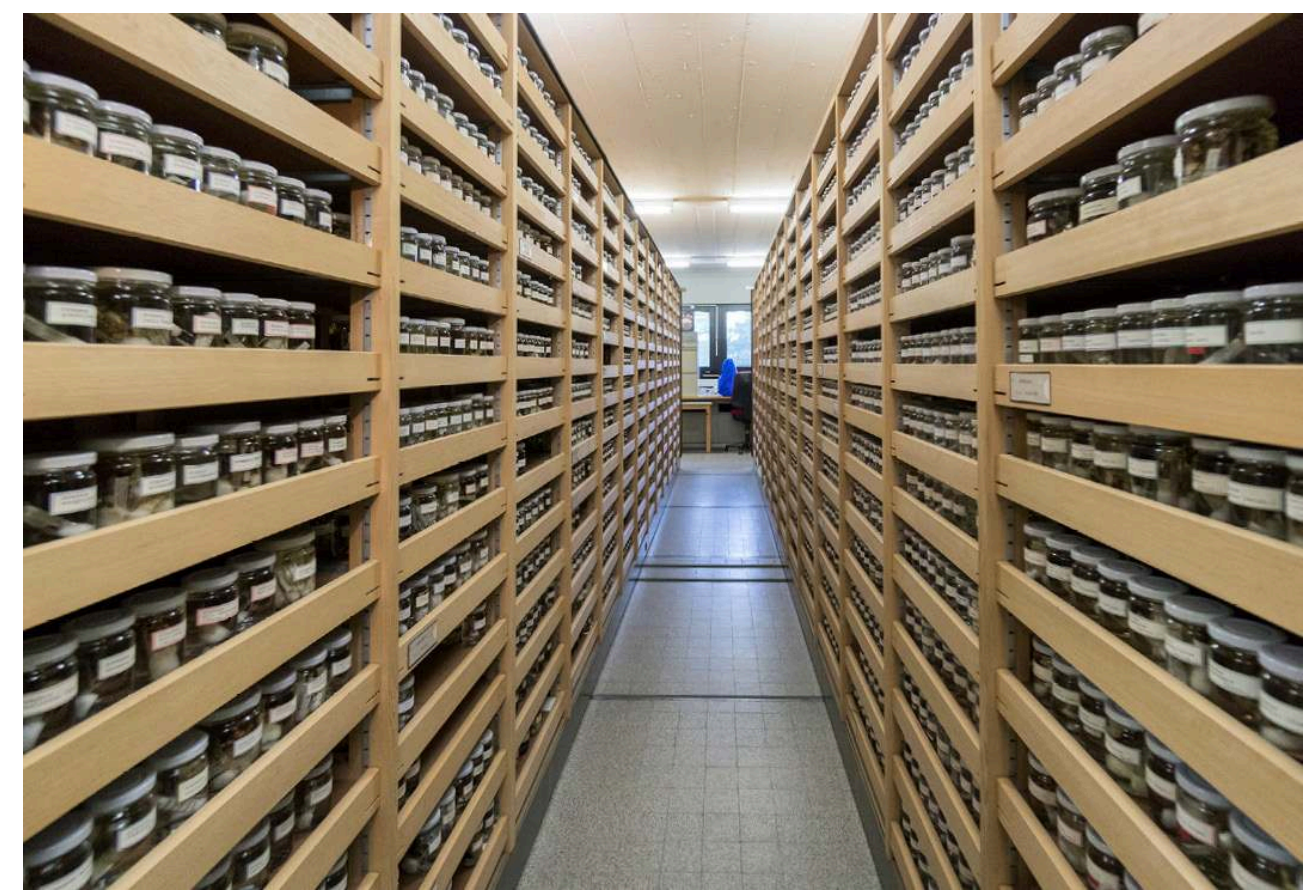

(c) P. Wagneur, MHNG

L'évaluation des retombées qualitatives du PSC apporte également de précieux enseignements.

Tout d'abord, un tel document a constitué jusqu'ici un outil précieux pour la direction qui doit en permanence prendre des décisions sur la pertinence des projets et des actions initiés, tout en gérant un quotidien envahissant. Ensuite, son utilisation a permis un dialogue constructif et une argumentation solide auprès des partenaires et des autorités. Le PSC a été aussi un excellent support de communication auprès des équipes. Cependant, un tel projet nouveau et inédit a bousculé les habitudes et les anciens schémas de fonctionnement ancrés depuis des décennies. Il s'est heurté notamment à la résistance au changement de certains collaborateurs qui n'adhéraient pas au concept écosystémique du PSC, préférant conserver une vision dichotomique du musée avec d'un côté les missions scientifiques et de l'autre, les activités culturelles. Enfin, le processus de mutation et sa marge de manœuvre ont été rendus difficiles par un contexte économique plus contraignant, rendant impossible la création de postes supplémentaires et l'augmentation du budget annuel alloué par la tutelle. 


\section{Discussion et recommandations}

27 Ces différentes expériences vécues durant l'élaboration et la mise en œuvre du PSC permettent aujourd'hui de tirer quelques leçons et proposer certaines recommandations utiles pour augmenter les chances de succès et le développement harmonieux d'un PSC.

Premièrement, l'élaboration d'un tel document doit rester dans une démarche résolument constructive en mettant avant tout l'accent sur les perspectives de développement tout en identifiant, sans jugement, les forces et les faiblesses de l'institution. Ceci est primordial afin de garantir la confiance et l'adhésion des équipes et, plus tard, leur pleine implication. Durant tout le proces $\neg$ sus, il faut veiller aussi à la participation de la majorité des équipes tout en évitant un dispersement d'idées pouvant amener à des frustrations.

On l'a vu, ce premier PSC a permis d'amorcer un certain nombre de mutations au sein de l'institution.

L'important maintenant est de maintenir le cap sur le moyen et le long terme. Dans cette optique, une nouvelle édition du PSC est prévue notamment pour accompagner au mieux le projet d'extension et de rénovation partielle du muséum et ses nouvelles perspectives.

31 En conclusion, le choix de l'élaboration d'un projet scientifique et culturel pour le muséum de Genève s'est avéré tout à fait adapté à la démarche de mutation de l'institution voulue par la tutelle. Cette dernière a validé et souligné l'importance et l'utilité de la démarche menée par le muséum de Genève. Il est d'ailleurs désormais demandé aux autres institutions muséales de la Ville de Genève d'établir également leur PSC. Pour ce qui est de la Suisse, avec ses 1100 musées - la plus forte concentration mondiale par habitant - l'offre muséale est certes foisonnante, mais cette densité exceptionnelle engendre également un problème de répartition des ressources dans un contexte économique toujours plus concurrentiel et désormais fortement mis à mal par la crise de Covid-19. Dans cette perspective, le développement d'une démarche comme celle du PSC pourrait vraisemblablement aider les institutions à clarifier leurs objectifs et leur rôle auprès des tutelles et des financeurs.

\section{BIBLIOGRAPHIE}

Ayer J. (dir). Muséum Genève. 200 ans d'histoire naturelle. Lausanne : Éditions Favre [à paraître].

Ayer J., Moeschler P., Heizmann P.-H., Mariaux J. et Pellegrini B. Projet scientifique et culturel "Muséum Genève 2020 ». Muséum d'histoire naturelle de la Ville de Genève, 2014 [en ligne].

Joly M.-H. Le Projet Scientifique et Culturel a-t-il de l'avenir ? La lettre de l'Ocim, nº124, juillet-août 2009, pp. 8-14

Mairesse F. Missions et évaluation des musées. Paris : L'Harmattan, 2004, 240 p. 
Le projet scientifique et culturel, Direction des musées de France : https://www.culture.gouv.fr/ content/download/57167/file/museofiche_PSC_20200623.pdf

\section{RÉSUMÉS}

Retour sur le processus et les modalités de la collecte participative entreprise par le musée des Civilisations de l'Europe et de la Méditerranée (Mucem) à Marseille pour documenter l'épidémie du Covid-19 et la situation inédite de confinement qui s'est ensuivie.

INDEX

Mots-clés : projet d'établissement (PSC) ; musée général

\section{AUTEUR}

\section{JACQUES AYER}

Muséologue et paléontologue. Il a notamment été à la tête du muséum de Genève de 2012 à 2020 .

ayerjacques@bluewin.ch 